\title{
Elastic effects of vacancies in strontium titanate: Short- and long-range strain fields, elastic dipole tensors, and chemical strain
}

\author{
Daniel A. Freedman, ${ }^{1,2}$ D. Roundy, ${ }^{3}$ and T.A. Arias ${ }^{1,2}$ \\ ${ }^{1}$ Laboratory of Atomic and Solid State Physics, Cornell University, Ithaca, New York 14853, USA \\ ${ }^{2}$ Cornell Center for Materials Research, Cornell University, Ithaca, New York 14853, USA \\ ${ }^{3}$ Department of Physics, Oregon State University, Corvallis, Oregon 97331, USA
}

(Dated: November 18, 2008)

\begin{abstract}
We present a study of the local strain effects associated with vacancy defects in strontium titanate and report the first calculations of elastic dipole tensors and chemical strains for point defects in perovskites. The combination of local and long-range results will enable determination of x-ray scattering signatures that can be compared with experiments. We find that the oxygen vacancy possesses a special property — a highly anisotropic elastic dipole tensor which almost vanishes upon averaging over all possible defect orientations. Moreover, through direct comparison with experimental measurements of chemical strain, we place constraints on the possible defects present in oxygen-poor strontium titanate and introduce a conjecture regarding the nature of the predominant defect in strontium-poor stoichiometries in samples grown via pulsed laser deposition. Finally, during the review process, we learned of recent experimental data, from strontium titanate films deposited via molecular-beam epitaxy, that show good agreement with our calculated value of the chemical strain associated with strontium vacancies.
\end{abstract}

PACS numbers: 61.72.Bb,61.72.Hh,61.72.jd,62.20.D-

\section{INTRODUCTION}

Perovskites in general, and strontium titanate in particular, are some of the most frequently and exhaustively studied materials in solid-state physics and chemistry. This attention has largely derived from their diverse and interesting properties: high piezoelectricity, 1 quantum paraelectricity, 23] ferroelectricity, ${ }^{4 / 7}$ uniaxial stress, $\frac{8}{8}$ and colossal magnetoresistance ${ }^{910}$ Further, the cubic perovskites manifest intriguing effects of underlying quantum fluctuations, since, although they are comprised of relatively heavy atomic constituents, a number of competing structures are energetically and structurally similar. ${ }^{11}$ Characterization of the low-temperature order parameters of these materials remains an open and engaging question. $\frac{12}{}$

Strontium titanate is a model perovskite - commonly available and reflective of many of the above properties of that material family. Specifically, while strontium titanate is a wide band-gap insulator at room temperature, it exhibits semiconductivity at elevated temperatures through doping or non-stoichiometric composition $13 \mid 14$ and superconductivity at low temperatures through reduction via addition of oxygen! $\frac{15 \mid 16}{}$ The structural phase diagram of strontium titanate comprises a high-temperature cubic phase and a low-temperature tetragonal phase, with a transition temperature near $105 \mathrm{~K} ! 17[19$ The cubic perovskite structure is particularly interesting due to the richness of its phase diagram (nonpolar antiferrodistortive to ferroelectric to antiferroelectric phases) ${ }^{20 \mid 21}$ to the capacity of these phases to emerge from miniscule deviations from the cubic lattice and its skeleton of octahedral oxygens (often through rigid rotations of such), and to open questions regarding the types (displacive or order-disorder) of the transitions among these phases.11
Defects and vacancies play a particularly important role in the chemistry of perovskites and deserve continued study in strontium titanate due to the electronic and superconducting effects of doping as well as their role in the interface region of heterostructures ${ }^{22}$ In the dilute limit, the mechanics of defects are fully determined by an examination of stress-strain effects, in particular the elastic dipole tensor, which motivates this work's emphasis on such a quantity. Our presentation of both short-range displacements around a point defect as well as long-range effects (characterized by the elastic dipole tensor) allows for the calculation of x-ray scattering signatures. These quantities also enable the prediction of defect mechanics, such as the behavior of defects within an externally imposed strain gradient (as present in heterostructures), as well as the ratio of chemical strain to stoichiometric deviation (a direct experimental observable). Finally, through comparison of our predictions of chemical strain with experimental results, we draw a number of important conclusions regarding the nature of point defects in non-stoichiometric strontium titanate.

\section{BACKGROUND}

Point defects introduce lattice distortions on both local and long-range scales. While the short-range distortions must be described by a potentially large set of atomic displacements, the long-range elastic distortions may be completely described by a single tensor, the elastic dipole tensor. 23

The elastic dipole tensor and its relation to elastic effects may be understood by the following simple considerations. Consider to quadratic order the most general expansion of the free energy per unit volume of a crys- 
tal in terms of the strain $\epsilon_{i j}$ ( $i$ and $j$ refer to coordinate axes) and the number of defects per unit volume $n_{d}$,

$$
\begin{aligned}
f\left(\epsilon_{i j}, n_{d}\right)=f_{0} & +n_{d} E_{d}+\frac{1}{2} n_{d}^{2} E_{d d} \\
& +\frac{1}{2} \sum_{i j k l} C_{i j k l} \epsilon_{i j} \epsilon_{k l}-n_{d} \sum_{i j} \epsilon_{i j} G_{i j} .
\end{aligned}
$$

The Taylor expansion coefficients $E_{d}, E_{d d}, C_{i j k l}$, and $G_{i j}$ are, respectively, the defect formation energy, an average inter-defect interaction energy, the components of the elastic stiffness tensor of the material, and the components of the elastic dipole tensor of the defects. The negative derivative of the free energy (1) with respect to strain then gives the stress,

$$
-\frac{\partial f}{\partial \epsilon_{i j}} \equiv \sigma_{i j}=-\sum_{k l} C_{i j k l} \epsilon_{k l}+n_{d} G_{i j} .
$$

To isolate the elastic dipole tensor, we can consider the rate of change of the stress in the crystal per unit concentration of defects, while holding strain fixed, that is, under strain control. Although challenging experimentally, strain control is quite convenient computationally since it corresponds to performing calculations with fixed lattice vectors. This derivative thus gives the elastic dipole tensor $\mathbf{G}$ directly,

$$
\left.\frac{\partial \sigma_{i j}}{\partial n_{d}}\right|_{\epsilon}=G_{i j},
$$

so that positive diagonal components of $\mathbf{G}$ indicate that the presence of defects tends to expand the crystal along the corresponding directions.

Alternatively, we can also consider the derivative of the strain in the crystal per unit concentration of defects under stress or load control (holding stress fixed). While stress control is computationally more complicated than strain control, it is the most common experimental situation. Under experimental conditions, the crystalline lattice vectors relax such that there is essentially zero stress (under normal laboratory conditions, atmospheric pressure corresponds to a negligible stress). This criterion allows the relation of strain to a newly defined quantity, $\Lambda$,

$$
\left.\frac{\partial \epsilon_{i j}}{\partial n_{d}}\right|_{\sigma}=\sum_{k l} S_{i j k l} G_{k l} \equiv \Lambda_{i j},
$$

where $\mathbf{S}$, the elastic compliance tensor, is the inverse of the elastic stiffness tensor $\mathbf{C}$, and $\boldsymbol{\Lambda}$ is defined as the defect-strain tensor, which is the strain per unit defect concentration induced in a crystal at fixed stress.

Relying upon the above derivations, the numerical calculation of the elastic dipole tensor $\mathbf{G}$ is straightforward. We compute the stress induced with the introduction of a single defect in a supercell (maintaining fixed lattice vectors, but allowing relaxation of the atomic coordinates). From Equation (3), this yields

$$
\begin{aligned}
G_{i j} & =\frac{1}{n_{d}}\left(\sigma_{i j}^{d}-\sigma_{i j}\right) \\
& =V_{\circ} \Delta \sigma_{i j},
\end{aligned}
$$

where $\sigma^{d}$ and $\sigma$ are the stresses with and without the defect in the cell, respectively, and $V_{\circ}$ is the supercell volume. Once $\mathbf{G}$ is known, $\boldsymbol{\Lambda}$ may also be computed directly from Equation (4). As a practical matter, we note that in this approach, the lattice vectors need not be those of a fully relaxed bulk crystal, provided the strain is small and kept fixed.

Experimental works often report the variations in chemical strain, the strain due to the presence of defects, with respect to stoichiometric deviations in the crystalline chemical formula. From the above considerations, the chemical strain is $\boldsymbol{\epsilon}_{c} \equiv n_{d} \boldsymbol{\Lambda}$. Deviations in stoichiometry specify the number of defects per chemical unit, $\delta$, so that, in this context, the concentration of defects per unit volume is $n_{d}=\delta / V_{c}$, where $V_{c}$ is the volume of the chemical unit. These two relations then immediately provide the chemical strain as proportional to this stoichiometric defect deviation, $\delta$,

$$
\boldsymbol{\epsilon}_{c}=\left(\frac{\boldsymbol{\Lambda}}{V_{c}}\right) \delta
$$

Experimentally, one does not generally obtain a full tensor for $\boldsymbol{\epsilon}_{c}$ but, instead, an average over all equivalent defect orientations which restore the symmetry of the underlying crystal. In cubic crystals, one measures a scalar $\epsilon_{c}$ which corresponds to the mean diagonal component of $\boldsymbol{\epsilon}_{c}$.

\section{METHODS}

To simulate strontium titanate, we employ a shell-potential mode ${ }^{24}$ parameterized for strontium titanate. 25 Shell-potential models are formulated as an extension to ionic pair potentials and employed to capture the polarizability of the atomic constituents. The shell model separates each ion into two parts, a core and an outer shell, which possess individual charges that sum to the nominal charge of the ion. The total model potential $U$ consists of three terms,

$$
U \equiv U_{P}+U_{C}+U_{B}
$$

representing, respectively, the polarizability of the ions and the Coulomb and short-range interactions among the ions. The polarizability is captured by harmonic springs connecting the core and shell of each ion, so that $U_{P}$ has the form,

$$
U_{P}=\frac{1}{2} \sum_{i} k_{i}\left|\Delta r_{i}\right|^{2}
$$

where $\left|\Delta r_{i}\right|$ is the core-shell separation for ion $i$ and the $k_{i}$ are a set of ion-specific spring constants. The Coulomb contributions take the form,

$$
U_{C}=\frac{1}{2} \sum_{i, j}^{\prime} \frac{k_{c} q_{i} q_{j}}{r_{i j}}
$$




\begin{tabular}{cccc}
\hline Ion & $\begin{array}{c}\text { Shell } \\
\text { Charge }[\mathrm{e}]\end{array}$ & $\begin{array}{c}\text { Core } \\
\text { Charge }[\mathrm{e}]\end{array}$ & $\begin{array}{c}\text { Spring Constant } \\
{\left[\mathrm{eV} \cdot \AA^{-2}\right]}\end{array}$ \\
\hline $\mathrm{Sr}^{2+}$ & 1.526 & 0.474 & 11.406 \\
$\mathrm{Ti}^{4+}$ & -35.863 & 39.863 & 65974.0 \\
$\mathrm{O}^{2-}$ & -2.389 & 0.389 & 18.41 \\
\hline
\end{tabular}

TABLE I: Electrostatic shell-model potential parameters for strontium titanate (from Akhtar et al. ${ }^{25}$ ).

\begin{tabular}{cccc}
\hline Interaction & $\mathrm{A}[\mathrm{eV}]$ & \multicolumn{1}{c}{$\rho[\AA]$} & $\mathrm{C}\left[\mathrm{eV} \cdot \AA^{6}\right]$ \\
\hline $\mathrm{Sr}^{2+} \Leftrightarrow \mathrm{O}^{2-}$ & 776.84 & 0.35867 & 0.0 \\
$\mathrm{Ti}^{4+} \Leftrightarrow \mathrm{O}^{2-}$ & 877.20 & 0.38096 & 9.0 \\
$\mathrm{O}^{2-} \Leftrightarrow \mathrm{O}^{2-}$ & 22764.3 & 0.1490 & 43.0 \\
\hline
\end{tabular}

TABLE II: Short-range shell-model potential parameters for strontium titanate (from Akhtar et al.25).

where $i$ and $j$ range over all cores and shells (excluding terms where $i$ and $j$ refer to the same ion), $q_{i}$ and $q_{j}$ are the corresponding charges, $r_{i j}$ is the distance between the charge centers, and $k_{c}$ is Coulomb's constant. We compute this Coulombic interaction ${ }^{26}$ using a Particle Mesh Ewald algorithm $27 \mid 29]$ with all real-space pairpotential terms computed out to a fixed cutoff distance using neighbor tables. Finally, the short-range interactions are included through a sum of Buckingham 30 pair potentials (which can be viewed as combinations of BornMayer $^{31}$ and Lennard-Jones ${ }^{32}$ potentials) of the form,

$$
U_{B}=\frac{1}{2} \sum_{i, j}\left(A_{i j} e^{-r_{i j} / \rho_{i j}}-C_{i j} r_{i j}^{-6}\right),
$$

where $i$ and $j$ range over all shells and $A_{i j}, \rho_{i j}$, and $C_{i j}$ are pair-specific adjustable parameters. Here, the first term (Born-Mayer) serves as a repulsive short-range interaction to respect the Pauli exclusion principle, and the second term (Lennard-Jones) models the dispersion or van der Waals interactions. ${ }^{33}$ The specific electrostatic and short-range shell-model parameters used in this study were fit to strontium titanate by Akhtar et al. $\frac{25}{}$ with values as listed in Tables II and II. Finally, we wish to emphasize again, as it is rarely mentioned explicitly in the shell-potential literature, that the pairpotential terms in $U_{B}$ apply to the shells only, and not to the cores.

Shell models have been extensively used for decades as the primary empirical potential for modeling perovskites and other oxides ${ }^{[3 / 35}$ We tested the correctness of our coded implementation of this potential through comparisons of lattice constants and elastic moduli and find excellent agreement. For instance, using the same shell potential and ground-state structure, we predict a lattice constant for cubic strontium titanate of $3.881 \AA$, which is within $0.3 \%$ of the value calculated by Akhtar et al. ${ }^{25}$ For the elastic moduli, we find $C_{11}=306.9 \mathrm{GPa}$, $C_{12}=138.7 \mathrm{GPa}$, and $C_{44}=138.8 \mathrm{GPa}$, which are within $1.8 \%, 1.0 \%$, and $0.7 \%$, respectively, of the values from Akhtar et al. ${ }^{25}$ From this, we conclude that our implementation of the potential is correct.

We further note that the static dielectric constant of 216.99 , as calculated by Akhtar et al ${ }^{25}$ for the same parameter set as our shell potential, is $28 \%$ lower than the experimental value for strontium titanate of 301.00 , a level of agreement typical of results from empirical potentials. As Gillan $\frac{36 / 37}{}$ and Stoneham ${ }^{38}$ discuss, there exists an important electrostriction effect whereby the tendency of the crystal to screen electric fields impacts the elastic dipole tensor. (Note that this effect scales as $1-\varepsilon^{-1}$, where $\varepsilon$ is the static dielectric constant ${ }^{37}$ ) Given the high dielectric constant of strontium titanate, this screening is nearly perfect in both the actual experiment and our model case; therefore, we expect that this effect is captured well in our calculations below, despite our relatively large fractional error in the static dielectric constant.

As is well known, strontium titanate has a large number of similar, competing ground-state structures. ${ }^{20} \mathrm{We}$ should emphasize, at this point, that the main quantities of interest to this study, either local atomic displacements or the elastic dipole and defect-strain tensors [from Equations (3)-(5)], are all defined as defect-induced changes relative to the bulk structure and so are likely quite insensitive to which of the competing structures are used to represent the bulk.

Accordingly, we have carried out what we regard as a thorough, but not exhaustive, search for a probable ground-state structure. Indeed, we have found no alternative structure which relaxes to an energy less than our candidate ground-state structure within our potential. We performed quenches on hundreds of random displacements from the idealized positions of the $1 \times 1 \times 1$ primitive unit cell to explore various potential reconstructions for supercells up to $6 \times 6 \times 6$. We also considered a number of highly ordered, human-inspired configurations commensurate with the antiferrodistortive disordering that is observed experimentally ${ }^{39 / 40}$ and predicted theoretically! ${ }^{20141 / 42}$ Among those minima which we explored, we selected the lowest-energy configuration to serve as the bulk crystalline state throughout this study. This configuration possesses a fairly regular pattern, namely, each oxygen octahedron rotates slightly along $\pm[111]$ (trigonal) directions in an alternating threedimensional $2 \times 2 \times 2$ checkerboard pattern. To aid in visualizing this reconstruction, Figure 1 depicts the atomic structure of reference non-reconstructed cubic strontium titanate, with the oxygen octahedra lattice indicative of perovskite materials.

We investigated five defects in strontium titanate: the oxygen, strontium, and titanium vacancies and the strontium-oxygen and titanium-oxygen divacancies. Since the octahedra rotations break the original crystalline symmetry and generate a set of different symmetry-related reconstructions, each of these five defects can be situated in multiple equivalent sites within each reconstruction. 


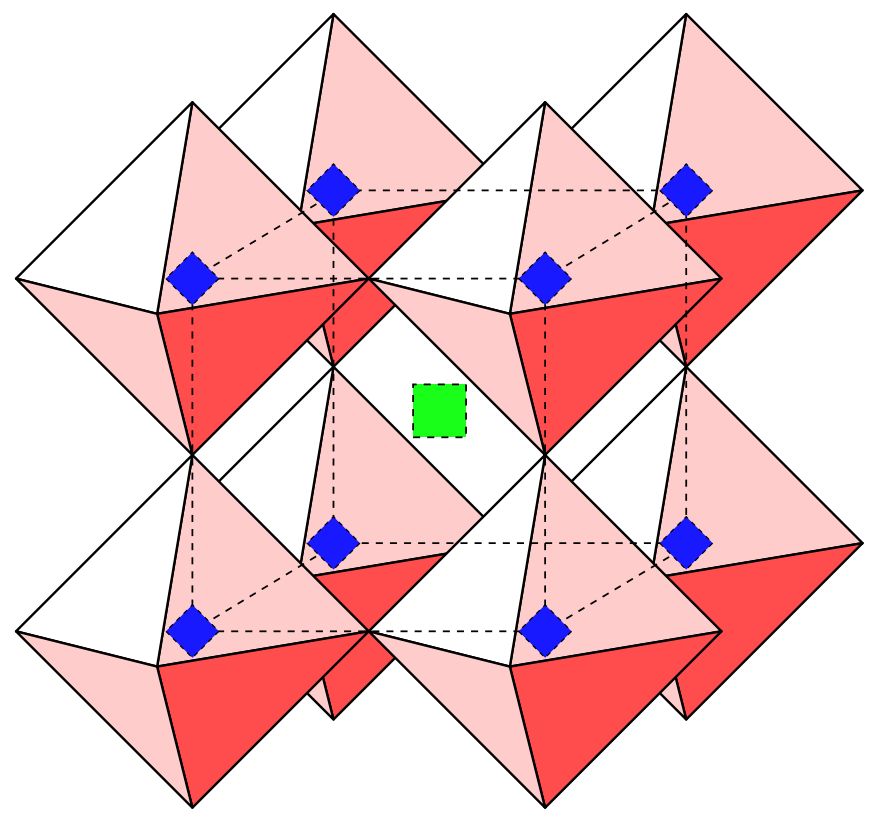

FIGURE 1: Atomic structure of non-reconstructed cubic strontium titanate: oxygen octahedra $(\circledast)$ surrounding titanium atoms $(\diamond)$, with strontium atoms $(\square)$ outside the octahedra and equidistant from the titanium sites.

Even well below room temperature, strontium titanate shows local fluctuations among the possible reconstructions. Thus, in addition to raw results for a specific realization of each defect in a given reconstruction, we also report results for each defect averaged over all possible reconstructions for a given orientation of the defect. The strontium and titanium vacancies do not select a specific direction and, thus, this averaging represents the full cubic crystalline symmetry group; their respective tensors therefore are always diagonal with cubic symmetry. The other defects do select specific crystalline directions, which must be specified when reporting the corresponding defect tensors. We thus reconstructionally average these latter defect tensors using either symmetry arguments or explicit calculations with different reconstructions, as appropriate. Finally, with a view to chemical strain measurements in macroscopic samples, we also report final averages over all defect orientations.

In the case of the oxygen vacancy, the oxygen sits between two unique nearest-neighbor titanium atoms, thus uniquely distinguishing the $\mathrm{Ti}-\mathrm{V}_{\mathrm{O}}-\mathrm{Ti}$ direction, which we define as [100], among the three cubic axes. Next, the titanium-oxygen divacancy selects a unique $\mathrm{V}_{\mathrm{Ti}}-\mathrm{V}_{\mathrm{O}}$ direction, which we define as [100], directed from the titanium site toward the oxygen site. Finally, the strontium-oxygen divacancy selects a unique $\mathrm{V}_{\mathrm{Sr}}-\mathrm{V}_{\mathrm{O}}$ direction, which we define as [11̄0], directed from the strontium toward the oxygen site. Once the reconstructional averaging is accomplished, the average over defect orientations requires generation of the crystal's response to all different possible orientations (three for the oxygen vacancies, six for the titanium-oxygen divacancy, and twelve for the strontium-oxygen divacancy) and restores full cubic symmetry.

To obtain the results in Section IV, each of these defects was placed within the bulk-reconstructed strontium titanate supercell, with cubic symmetry as experimentally observed for $T \gtrsim 105 \mathrm{~K}, \frac{17-19}{19}$ and then relaxed via the technique of preconditioned conjugate gradient minimization ${ }^{43}$ (specifically, the Polak-Ribière ${ }^{44}$ method) to find the minimal energy configuration (to within $\sim 1 \mu \mathrm{eV}$ ). Supercell convergence studies examined all five defects in cells containing up to 13720 atoms and verified that such defects were sufficiently separated so that interactions across periodic supercell boundaries were negligible ${ }^{45}$ The final relaxed atomic configurations in the largest cells $(14 \times 14 \times 14)$ provide the local strain fields which we report below for each defect. To determine the long-range strain fields, we compute the elastic dipole tensor $\mathbf{G}$ through the prescription described in Equation (5) above; namely, we calculate the stress induced by the introduction of a single defect in the supercell, holding the lattice vectors fixed while allowing the atomic coordinates to relax.

\section{RESULTS}

For a series of important and fundamental strontium titanate defects, we examine both the elastic dipole and defect-strain tensors, as introduced in Section [II] as well as the local strains surrounding each defect. This section first examines the role of the oxygen vacancy as a case study of a defect in strontium titanate. Subsequently, the results for the same set of studies are presented for four other point defects in strontium titanate: strontium and titanium vacancies and strontium-oxygen and titanium-oxygen divacancies. Section $\mathrm{V}$ continues with more general implications of our results.

\section{A. Oxygen vacancy}

As described above, there are three distinct orientations for the oxygen vacancy as defined by the $\mathrm{Ti}-\mathrm{V}_{\mathrm{O}}-\mathrm{Ti}$ direction. Moreover, because of the reconstruction, there are in fact two distinct classes of site within each possible orientation. As one moves in the positive sense of direction along the $\mathrm{Ti}-\mathrm{V}_{\mathrm{O}}-\mathrm{Ti}$ axis, these sites are distinguished by whether the rotation of the octahedra surrounding the two titanium sites changes from positive to negative or from negative to positive (in the right-hand sense about the $+[111]$ direction). Below, we report results for the latter type of site.

We first examine the elastic dipole tensor computed according to Equation (5). To explore convergence, we compute the dipole tensor components in supercells of sizes $2 \times 2 \times 2,4 \times 4 \times 4, \ldots, 14 \times 14 \times 14$, containing between 40 and 13720 atoms with defect separations 


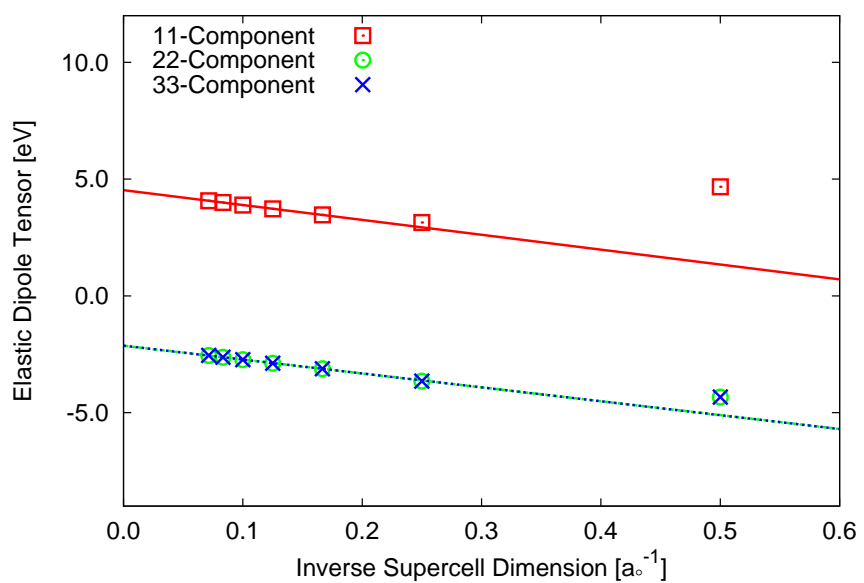

FIGURE 2: Diagonal components of elastic dipole tensor for oxygen vacancy in strontium titanate. Data show linear convergence with inverse linear dimension of supercell size.

between $\sim 8$ and $\sim 54 \AA$.

Figure 2 depicts the convergence of the diagonal components of the elastic dipole tensor as a function of inverse linear dimension of the supercell. The linear behavior in the figure for large cells indicates that this quantity converges in the same way as the Coulomb interaction between defects and allows extrapolation of the fully converged value for these components in an infinite supercell. We observe exactly the same linear behavior with inverse linear dimension of cell for the convergence of the off-diagonal components of the dipole tensor. A linear fit to the data, for all tensor components for cell sizes in the range exhibiting linear behavior $(6 \times 6 \times 6$ through $14 \times 14 \times 14$ ), yields the elastic dipole tensor, extrapolated to infinite cell size, for this oxygen vacancy in strontium titanate,

$$
\mathbf{G}_{\mathrm{O}}=\left(\begin{array}{rrr}
4.53 & -3.11 & -3.11 \\
-3.11 & -2.13 & 1.06 \\
-3.11 & 1.06 & -2.13
\end{array}\right) \mathrm{eV} .
$$

As described above in Section [II] at finite temperatures $(T \gtrsim 105 \mathrm{~K})$, local fluctuations in the reconstruction make it appropriate to average this tensor over all reconstructions. The result is to eliminate the off-diagonal components, leaving the reconstructionally averaged elastic dipole tensor,

$$
\overline{\mathbf{G}}_{\mathrm{O}}=\left(\begin{array}{rrr}
4.53 & 0.00 & 0.00 \\
0.00 & -2.13 & 0.00 \\
0.00 & 0.00 & -2.13
\end{array}\right) \mathrm{eV} .
$$

Since the underlying, non-defected, crystal is now cubic, we can readily apply Equation (4) to $\overline{\mathbf{G}}_{\mathrm{O}}$ to obtain the reconstructionally averaged defect-strain tensor,

$$
\overline{\boldsymbol{\Lambda}}_{\mathrm{O}}=\left(\begin{array}{rrr}
16.33 & 0.00 & 0.00 \\
0.00 & -8.05 & 0.00 \\
0.00 & 0.00 & -8.05
\end{array}\right) \AA^{3} .
$$

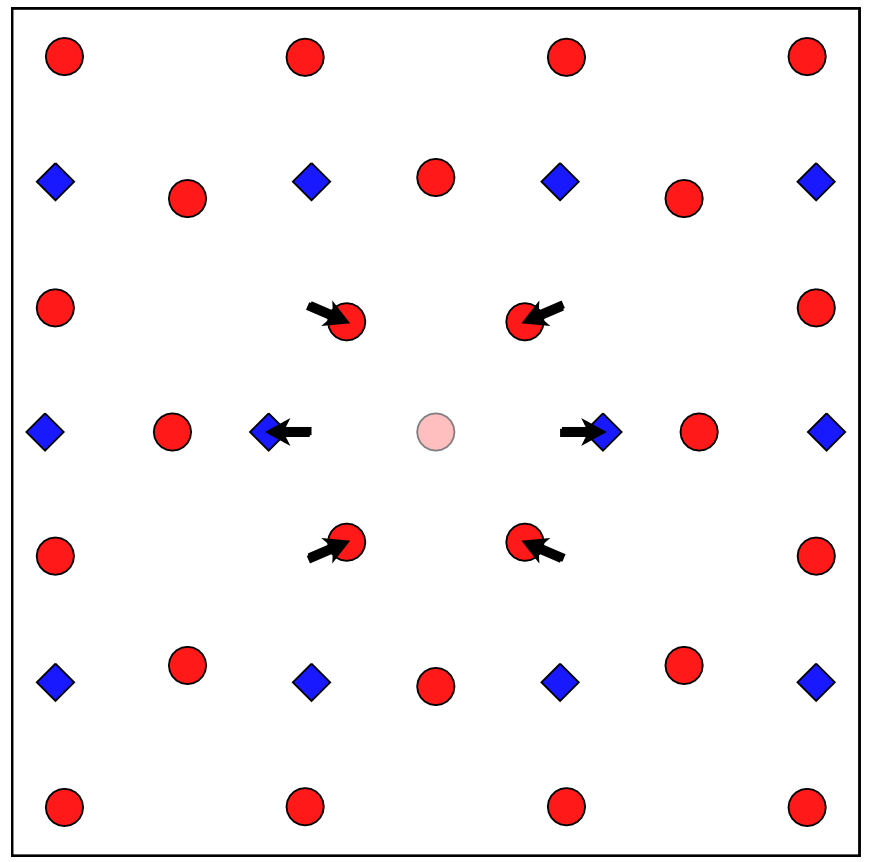

FIGURE 3: Local strain pattern for oxygen vacancy in the $\hat{e}_{1} \hat{e}_{2}$ plane of strontium titanate: titanium atoms $(\diamond)$, oxygen atoms $(\bigcirc)$, oxygen vacancy $(\bigcirc)$. Atomic displacements exaggerated by a factor of three for clarity $(\rightarrow)$, displayed for significant in-plane displacements $(>0.1 \AA)$ only.

The above results indicate that the oxygen vacancy tends to cause the crystal to expand along the $\mathrm{Ti}-\mathrm{V}_{\mathrm{O}}-\mathrm{Ti}$ direction and to contract along the two orthogonal directions by an amount which results in negligible net volume change in the crystal. When the above tensor is averaged over all defect orientations (permutations of the three coordinate axes), the result is near perfect cancellation, resulting in a constant tensor (multiple of the identity) with a uniform chemical strain per unit defect concentration of $+0.07 \AA^{3}$. This corresponds to a ratio of chemical strain $\epsilon_{c}$ to the deviation from stoichiometry $\delta$ in $\mathrm{SrTiO}_{3-\delta}$ of $\epsilon_{c} / \delta=+0.001$, indicating a very slight tendency for the crystal to expand due to the presence of oxygen vacancies.

Following Figure 3, we now examine the local strain of this system after reconstructional averaging. We note that the removal of the oxygen ion, with a nominal charge of -2 , leaves an effective local positive environment in the location of the vacancy. We should expect pronounced Coulombic response to this environment in the form of local crystal polarization. Indeed, the nearest neighbors of the oxygen vacancy, the two titanium atoms, now move directly away from the vacancy on the precise vector connecting them, by $0.21 \AA$. The next-nearest neighbors are the eight oxygens comprised of two equidistant rings of four oxygens each, each of which move $0.21 \AA$ toward the vacancy $\left(0.19 \AA\right.$ along $\hat{e}_{1}$ with remaining projection of $0.08 \AA$ either along $\hat{e}_{2}$ or $\hat{e}_{3}$ as dictated by symmetry), and one ring of four strontium atoms, each of which 


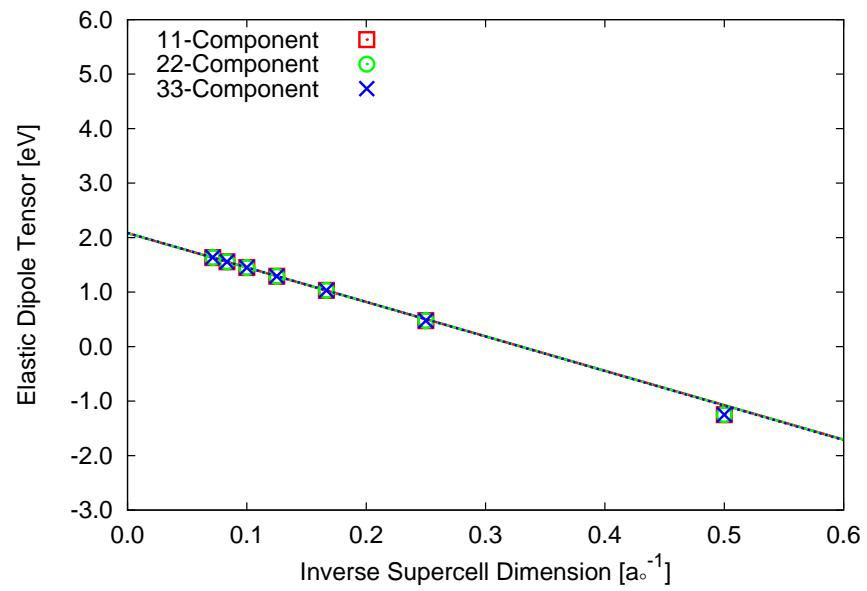

FIGURE 4: Diagonal components of elastic dipole tensor for strontium vacancy in strontium titanate. Data show linear convergence with inverse linear dimension of supercell size.

moves $0.10 \AA$ directly away from the vacancy. The third shell of neighbors, a set of six oxygens, each one lattice constant away from the vacancy along the three lattice directions in the crystal, moves different amounts depending upon the vector; the two oxygens along the $\mathrm{Ti}-\mathrm{V}_{\mathrm{O}}$-Ti direction move $0.06 \AA$ directly away from the vacancy (pushed sterically by the second-shell titanium atoms), while the other four oxygens move by a negligible amount (only $0.01 \AA$ ). While the fourth shell of neighbors, a set of eight titanium atoms at a distance $\sqrt{5} / 2 a_{\circ}$ from the vacancy, moves negligibly, the fifth shell of neighbors (sixteen oxygen and eight equidistant strontium atoms) shows significant movement in the strontium atoms of $0.15 \AA$ away from the vacancy $(0.08 \AA$ along $\hat{e}_{1}$ with remaining projections of $0.09 \AA$ along both $\hat{e}_{2}$ and $\hat{e}_{3}$ ), even though the oxygen atoms move a negligible amount. Finally, in the sixth shell of neighbors, a set of twelve oxygen atoms, eight oxygens (those not in the plane containing the defect and perpendicular to $\hat{e}_{1}$ ) move $0.10 \AA$ toward the vacancy $\left(0.02 \AA\right.$ along $\hat{e}_{1}$ with remaining projection of $0.10 \AA$ either along $\hat{e}_{2}$ or $\hat{e}_{3}$ as dictated by the symmetry), while four others (those in the plane perpendicular to $\hat{e}_{1}$ ) move a negligible amount. All other atoms in the crystal move less than $0.06 \AA$.

Finally, we would like to comment on the relation between local strains and defect tensors. We observe that the direction of motion of the near-neighboring atoms often correlates with the far-field motion described by the defect-strain tensor. In this case, in the first and second shells, we see a general pattern of movement which is away from the defect along $\hat{e}_{1}$ and toward the defect in the other two directions, consistent with the signs in the long-range defect-strain tensor.

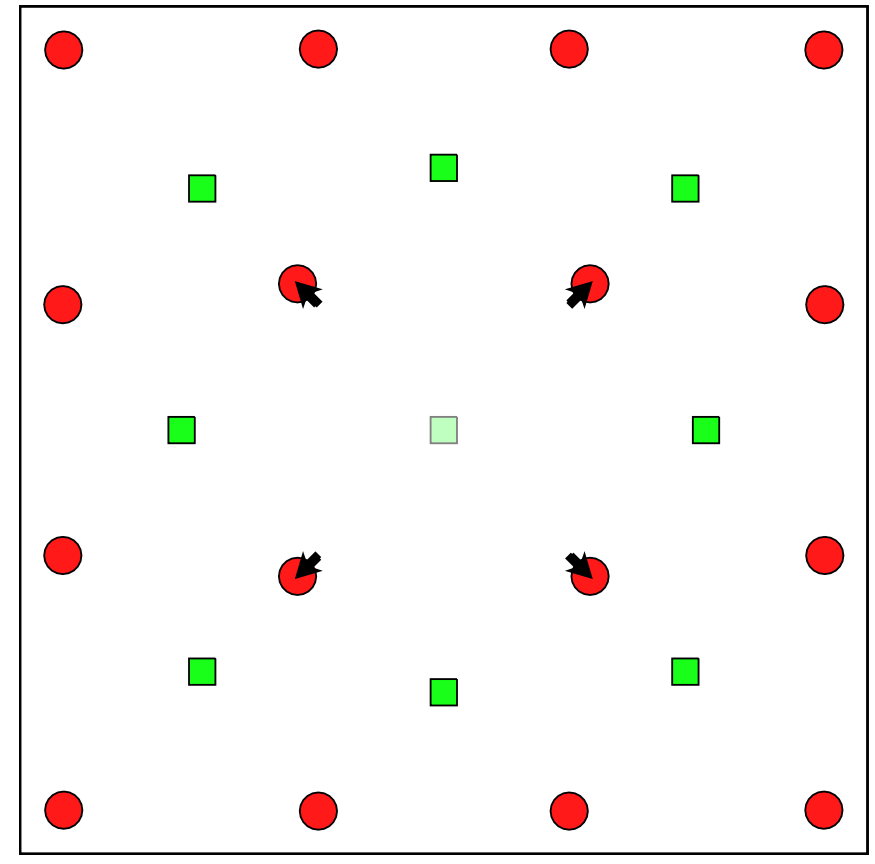

FIGURE 5: Local strain pattern for strontium vacancy in the $\hat{e}_{1} \hat{e}_{2}$ plane of strontium titanate: strontium atoms $(\square)$, oxygen atoms $(\bigcirc)$, strontium vacancy $(\square)$. Atomic displacements exaggerated by a factor of three for clarity $(\rightarrow)$, displayed for significant in-plane displacements $(>0.1 \AA)$ only.

\section{B. Strontium vacancy}

We now repeat the above procedures to obtain similar results for the strontium vacancy. As described above, the strontium-vacancy site defines no unique direction and reconstructional averaging recovers the full cubic symmetry group. For any realization of the reconstruction there are in fact two distinct types of strontium sites. Each such site sits at the center of a cube with oxygen octahedra at the corners with alternating signs of rotations. The results reported below, prior to reconstructional averaging, correspond to the site in which the rotation at the [111] corner is positive.

For the elastic dipole tensor we find

$$
\mathbf{G}_{\mathrm{Sr}}=\left(\begin{array}{rrr}
2.08 & -0.23 & -0.23 \\
-0.23 & 2.08 & -0.23 \\
-0.23 & -0.23 & 2.08
\end{array}\right) \mathrm{eV}
$$

where Figure 4 shows the convergence of the diagonal elements of the above tensor. Performing the reconstructional average gives

$$
\overline{\mathbf{G}}_{\mathrm{Sr}}=\left(\begin{array}{ccc}
2.08 & 0.00 & 0.00 \\
0.00 & 2.08 & 0.00 \\
0.00 & 0.00 & 2.08
\end{array}\right) \mathrm{eV},
$$

with a corresponding defect-strain tensor,

$$
\bar{\Lambda}_{\mathrm{Sr}}=\left(\begin{array}{lll}
1.78 & 0.00 & 0.00 \\
0.00 & 1.78 & 0.00 \\
0.00 & 0.00 & 1.78
\end{array}\right) \AA^{3} .
$$




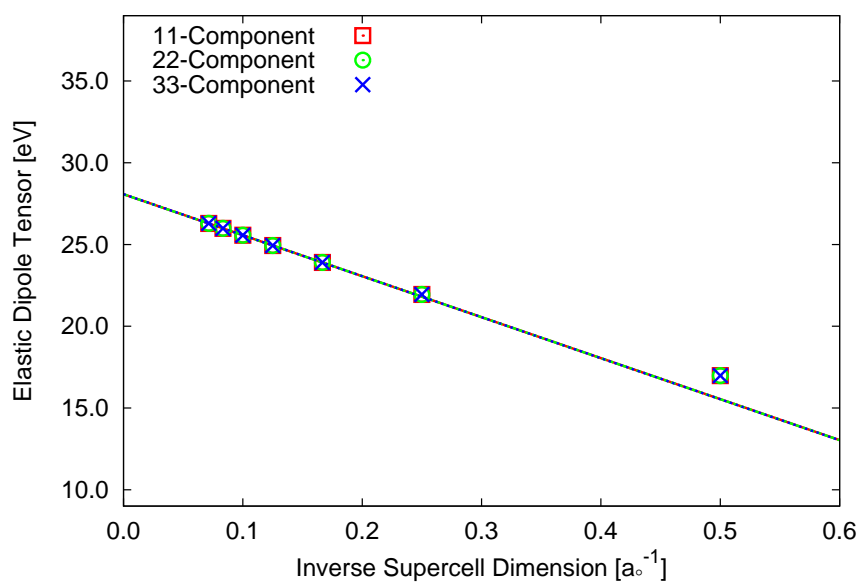

FIGURE 6: Diagonal components of elastic dipole tensor for titanium vacancy in strontium titanate. Data show linear convergence with inverse linear dimension of supercell size.

This result expresses the tendency of the crystal to expand due to the strontium vacancy, by an amount significantly greater than the net effect of the oxygen vacancy. Since the original defect defines no unique direction, no orientational averaging is necessary, and we find a ratio of chemical strain $\epsilon_{c}$ to the deviation from stoichiometry $\delta$ in $\mathrm{Sr}_{1-\delta} \mathrm{TiO}_{3}$ of $\epsilon_{c} / \delta=+0.030$, indicating a tendency for the crystal to expand due to the presence of strontium vacancies.

We now examine the local strains around such a strontium vacancy after reconstructional averaging (Figure 5). Twelve oxygen atoms (three in each of four neighboring octahedra) are nearest neighbors to the strontium vacancy; these twelve atoms all move directly away from the vacancy by a distance of $0.15 \AA$. The next-nearest neighbors are the eight titanium atoms in each of the surrounding octahedra, each of which moves $0.09 \AA$ directly toward the strontium vacancy. All other atoms move less than $0.08 \AA$.

In this case, as expected, both the defect-strain tensor and the local strain displacements are symmetric after reconstructional averaging. The nearest-neighbor displacement shows an expansion in all directions, similar to the defect-strain tensor. The next-nearest neighbors, which have an opposite charge from that of the nearest neighbors, move in the opposite direction, reinforcing that there is no simple connection between local displacements and far-field strain patterns.

\section{Titanium vacancy}

The titanium-vacancy site also defines no unique direction in the ideal crystal, and the reconstructional averaging restores the full cubic symmetry. For any realization of the reconstruction there are, in fact, two distinct types of titanium site. Each such site sits at the center of an octahedron with either positive or negative signs of the

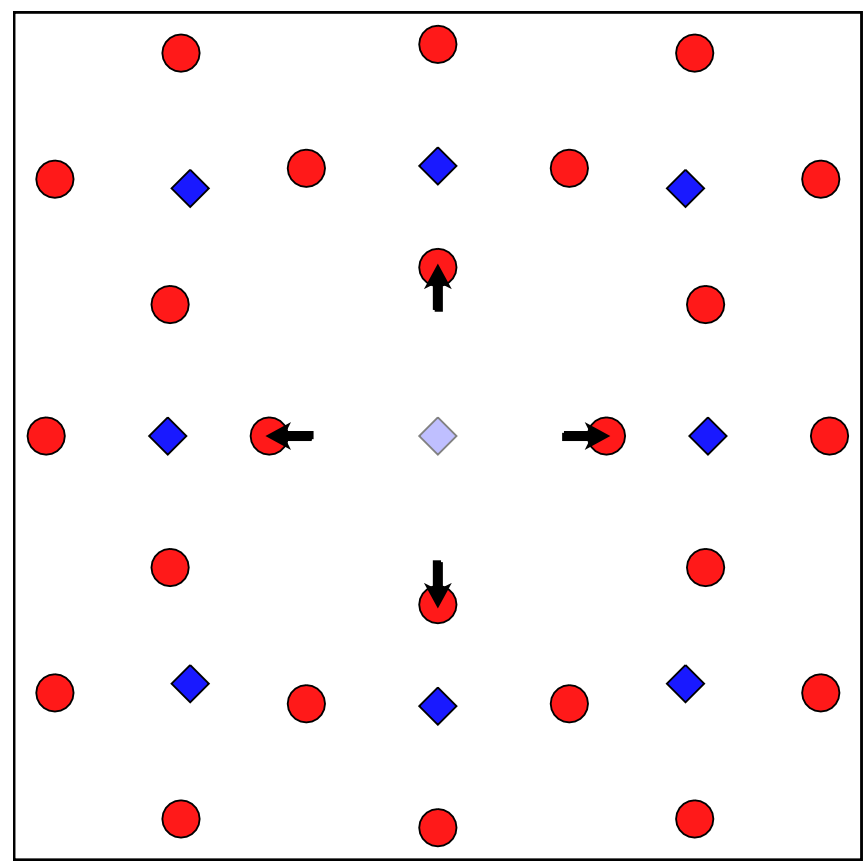

FIGURE 7: Local strain pattern for titanium vacancy in the $\hat{e}_{1} \hat{e}_{2}$ plane of strontium titanate: titanium atoms $(\diamond)$, oxygen atoms $(\bigcirc)$, titanium vacancy $(\diamond)$. Atomic displacements exaggerated by a factor of three for clarity $(\rightarrow)$, displayed for significant in-plane displacements $(>0.1 \AA)$ only.

rotations relative to the $+[111]$ axis. The results reported below, prior to reconstructional averaging, correspond to the site in which the rotation is positive.

We first report our results for the elastic dipole tensor,

$$
\mathbf{G}_{\mathrm{Ti}}=\left(\begin{array}{rrr}
28.08 & -0.70 & -0.70 \\
-0.70 & 28.08 & -0.70 \\
-0.70 & -0.70 & 28.08
\end{array}\right) \mathrm{eV},
$$

where Figure 6 shows the convergence of the diagonal elements of the above tensor. The reconstructional average is then

$$
\overline{\mathbf{G}}_{\mathrm{Ti}}=\left(\begin{array}{rrr}
28.08 & 0.00 & 0.00 \\
0.00 & 28.08 & 0.00 \\
0.00 & 0.00 & 28.08
\end{array}\right) \mathrm{eV},
$$

with defect-strain tensor,

$$
\overline{\boldsymbol{\Lambda}}_{\mathrm{Ti}}=\left(\begin{array}{rrr}
23.92 & 0.00 & 0.00 \\
0.00 & 23.92 & 0.00 \\
0.00 & 0.00 & 23.92
\end{array}\right) \AA^{3} .
$$

This defect-strain tensor expresses the tendency of the crystal to expand due to the titanium vacancy - a significantly greater amount even than that of the strontium. Again, since the original defect defines no unique direction, no orientational averaging over defect types is necessary. Finally, we report a ratio of chemical strain $\epsilon_{c}$ to the deviation from stoichiometry $\delta$ in $\mathrm{SrTi}_{1-\delta} \mathrm{O}_{3}$ of $\epsilon_{c} / \delta=+0.402$, indicating a significant tendency for 
the crystal to expand due to the presence of titanium vacancies.

As depicted in Figure 7, we now describe the local strain effects on the atoms surrounding the titanium vacancy. The nearest neighbors are six surrounding oxygen atoms which move $0.22 \AA$ directly away from the titanium vacancy. The next-nearest neighbors are the eight surrounding strontium atoms along the body diagonals from the titanium vacancies; these strontium atoms move the very significant distance of $0.52 \AA$ directly toward the titanium vacancy. The third shell is made up of six titanium atoms separated by a lattice constant from the vacancy along all three directions (positive and negative); all of these titanium atoms move $0.09 \AA$ directly away from the titanium vacancy. Finally, the fourth shell of atoms is comprised of twenty-four oxygen atoms, arranged as six groups of four oxygens, each in a diamondshape with its center one lattice coordinate away from the titanium vacancy in each lattice direction. These oxygens each move $0.08 \AA$ away from the vacancy $(0.08 \AA$ along the vector separating the diamond-group from the vacancy, and $0.03 \AA$ along either of the two other directions, so as to cause the diamond-group to spread outward). All other atoms in the crystal move less than $0.07 \AA$.

We again observe connections between the reconstructionally averaged local displacements and the far-field defect-strain tensor which have similar symmetry. In this case of the titanium vacancy, the nearest-neighbor atoms are displaced away from the vacancy, showing the same behavior as the defect-strain tensor. Interestingly, however, the next-nearest neighbors, which move toward the titanium vacancy, actually have more than twice the displacement of the nearest neighbors. So here we observe that the far-field strain does not correlate with the largest magnitude displacement, but instead with that of the nearest-neighbor atoms.

\section{Strontium-oxygen divacancy}

As described above in Section III, there are twelve distinct orientations for the strontium-oxygen divacancy as defined by the $\mathrm{V}_{\mathrm{Sr}}-\mathrm{V}_{\mathrm{O}}$ direction. For any realization of the reconstruction there are in fact two distinct types of strontium-oxygen sites. Each such strontium site sits at the center of a cube with oxygen octahedra at the corners with alternating signs of rotations. The results reported below, prior to reconstructional averaging, correspond to the $[1 \overline{1} 0]$ defect with negative sense of rotation for the octahedron at the [111] corner.

The elastic dipole tensor for this defect is

$$
\mathbf{G}_{\mathrm{SrO}}=\left(\begin{array}{rrr}
-4.62 & 3.00 & -2.28 \\
3.00 & -4.62 & -2.28 \\
-2.28 & -2.28 & 6.95
\end{array}\right) \mathrm{eV}
$$

where Figure 8 shows the convergence of the diagonal elements of the above tensor. The reconstructional average is then

$$
\overline{\mathbf{G}}_{\mathrm{SrO}}=\left(\begin{array}{rrr}
-3.00 & 1.78 & 0.00 \\
1.78 & -3.00 & 0.00 \\
0.00 & 0.00 & 4.27
\end{array}\right) \mathrm{eV},
$$

with defect-strain tensor,

$$
\overline{\boldsymbol{\Lambda}}_{\mathrm{SrO}}=\left(\begin{array}{rrr}
-9.37 & 2.14 & 0.00 \\
2.14 & -9.37 & 0.00 \\
0.00 & 0.00 & 17.25
\end{array}\right) \AA^{3} .
$$

This defect-strain tensor expresses a slight tendency of the crystal to contract due to the strontium-oxygen divacancy. The lower symmetry of this defect, with its orientation along a diagonal, leads to remaining off-diagonal elements even after reconstructional averaging. However, when the above tensor is averaged over all twelve defect orientations, the result is a constant tensor with a uniform chemical strain per unit concentration of defect of $-0.50 \AA^{3}$. This corresponds to a ratio of chemical strain $\epsilon_{c}$ to the deviation from stoichiometry $\delta$ in $\mathrm{Sr}_{1-\delta} \mathrm{TiO}_{3-\delta}$ of $\epsilon_{c} / \delta=-0.008$, indicating a tendency for the crystal to contract due to the presence of strontium-oxygen divacancies.

Referring to Figure 9, we now examine the local displacements after reconstructional averaging. The situation with this divacancy is more complicated than that of earlier isolated atomic vacancies, leading us to characterize the atomic displacements with respect to each independent missing atom in the strontium-oxygen divacancy. The first shell is comprised of the eleven remaining oxygen atoms that are nearest neighbors of the strontium vacancy. This strontium vacancy would normally have twelve neighboring oxygen atoms forming three squares in the three planes, each comprised of four atoms centered around the strontium vacancy; however, one of these oxygen atoms is missing to form the divacancy. The four oxygens, which are farthest from the oxygen vacancy but not in the same square as the oxygen vacancy, all move away from the strontium vacancy by $0.14 \AA(0.10 \AA$ along $\hat{e}_{3}$, and $0.09 \AA$ along either $\hat{e}_{1}$ or $\hat{e}_{2}$, depending upon symmetry, with $0.004 \AA$ along the opposite vector, chosen such that most of each displacement is within the plane of squares to which that oxygen belongs). The four oxygens that are closest to the oxygen vacancy and still not in the same square as the oxygen vacancy also move as a group. These oxygen atoms all move away from the strontium vacancy by $0.11 \AA\left(0.05 \AA\right.$ along $\hat{e}_{3}$, and $0.09 \AA$ along either $\hat{e}_{1}$ or $\hat{e}_{2}$, depending upon symmetry, with $0.05 \AA$ along the opposite vector, chosen such that more of each displacement is within the plane of the square to which that oxygen belongs). The two oxygens, in the same square as the oxygen vacancy and closer to such vacancy, are displaced away from the defect, in that plane, by $0.12 \AA\left(0.12 \AA\right.$ along either $\hat{e}_{1}$ or $\hat{e}_{2}$, and $0.04 \AA$ along the opposite vector, chosen to ensure that the displacement maximizes its overall movement away from the remaining oxygen in this square). The final oxygen atom 


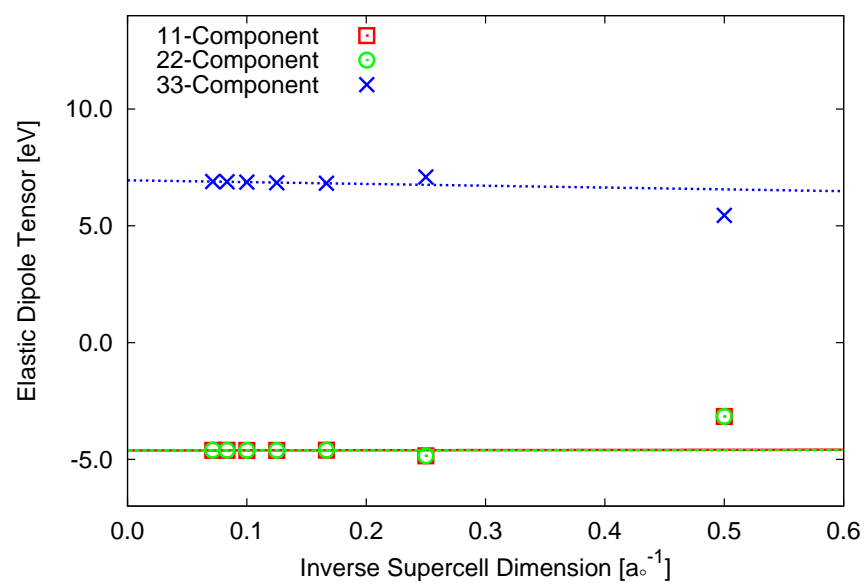

FIGURE 8: Diagonal components of elastic dipole tensor for strontium-oxygen divacancy in strontium titanate. Data show linear convergence with inverse linear dimension of supercell size.

is also in the same square as the oxygen vacancy, but is the farthest atom from the vacancy in this square; it is displaced directly away from the defect in that plane by $0.14 \AA$. Also included in the first shell are the remaining oxygen atoms that are nearest neighbors to the missing oxygen vacancy. That oxygen vacancy, part of two oxygen octahedra, has eight neighboring oxygens, four in each octahedron; of those eight oxygens, the four closest to the strontium vacancy have already been considered as part of the nearest neighbors to the missing strontium atom. The remaining four oxygen atoms in the other octahedron, farthest from the strontium vacancy, move the appreciable distance of $0.33 \AA$ toward the vector connecting the strontium and oxygen vacancies $\left(0.30 \AA\right.$ along $\hat{e}_{3}$, and $0.11 \AA$ along either $\hat{e}_{1}$ or $\hat{e}_{2}$, depending upon symmetry, with $0.005 \AA$ along the opposite vector, chosen such that most of each displacement in the $\hat{e}_{1} \hat{e}_{2}$ plane is toward the oxygen vacancy). Finally, this first shell also includes the three strontium atoms (the fourth is itself missing) nearest the oxygen vacancy. The one strontium atom farthest from the strontium vacancy moves directly toward the oxygen vacancy by $0.03 \AA(0.02 \AA$ along both $\hat{e}_{1}$ and $\left.\hat{e}_{2}\right)$. The other two strontium atoms move away from the oxygen vacancy by $0.10 \AA(0.10 \AA$ along either $\hat{e}_{1}$ or $\hat{e}_{2}$, depending upon symmetry, with $0.04 \AA$ along the opposite vector, chosen such that the displacement maximizes the distances of these strontium atoms from the strontium vacancy).

The second shell is comprised of the eight titanium atoms that are the nearest neighbors to the missing strontium atom. Two of these nearest neighboring titanium atoms, which could alternatively have been categorized as the nearest-neighboring shell of atoms from the oxygen vacancy, move away from the strontium vacancy (as well as the oxygen vacancy) by $0.19 \AA$ ( $0.19 \AA$ away from the vacancies along $\hat{e}_{3}$, and $0.03 \AA$ toward the strontium vacancy along both $\hat{e}_{1}$ and $\hat{e}_{2}$ ). Two other nearest-neighbor

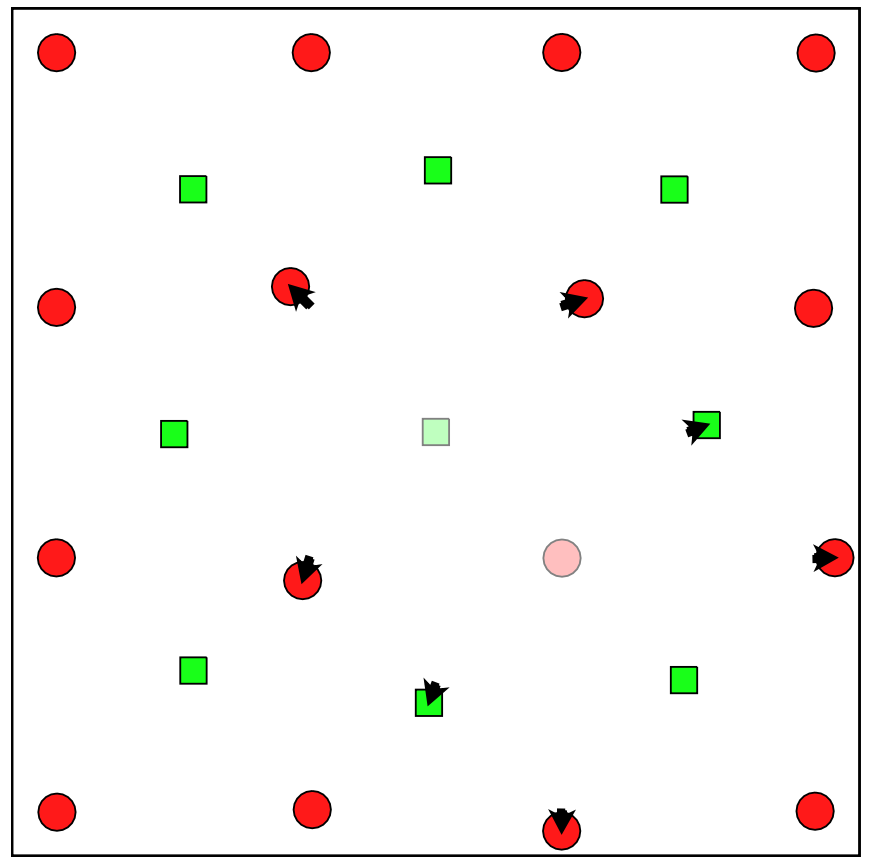

FIGURE 9: Local strain pattern for strontium-oxygen divacancy in the $\hat{e}_{1} \hat{e}_{2}$ plane of strontium titanate: strontium atoms $(\square)$, oxygen atoms $(\bigcirc)$, strontium vacancy $(\square)$, oxygen vacancy $(\bigcirc)$. Atomic displacements exaggerated by a factor of three for clarity $(\rightarrow)$, displayed for significant inplane displacements $(>0.1 \AA)$ only.

titanium atoms, those that are furthest from the oxygen vacancy among these eight titanium atoms, move $0.09 \AA$ toward the strontium vacancy $\left(0.06 \AA\right.$ along $\hat{e}_{3}$, and $0.05 \AA$ along both $\hat{e}_{1}$ and $\hat{e}_{2}$ ). The final four of these eight nearest-neighbor titanium atoms move toward the vacancies by $0.11 \AA\left(0.05 \AA\right.$ along $\hat{e}_{3}$, and $0.08 \AA$ along either $\hat{e}_{1}$ or $\hat{e}_{2}$, depending upon symmetry, with $0.05 \AA$ along the opposite vector, chosen such that more of each displacement in the $\hat{e}_{1} \hat{e}_{2}$ plane is toward the oxygen vacancy). All other atoms move less than $0.17 \AA$ A.

We now investigate correlations between these above reconstructionally averaged local displacements and the far-field defect-strain tensor. We find surprising results in this case of the strontium-oxygen divacancy. While the defect-strain tensor and local displacement pattern both show expansion on the [001] axis outward from the defect, we see a disagreement in sign in the (001) plane between the far-field contraction and the expansion of the nearest neighbors. This serves as a cautionary note that far-field and near-field strain patterns need not be simply related and reinforces the importance of providing both sets of information for further experimental analysis of x-ray scattering signatures. 


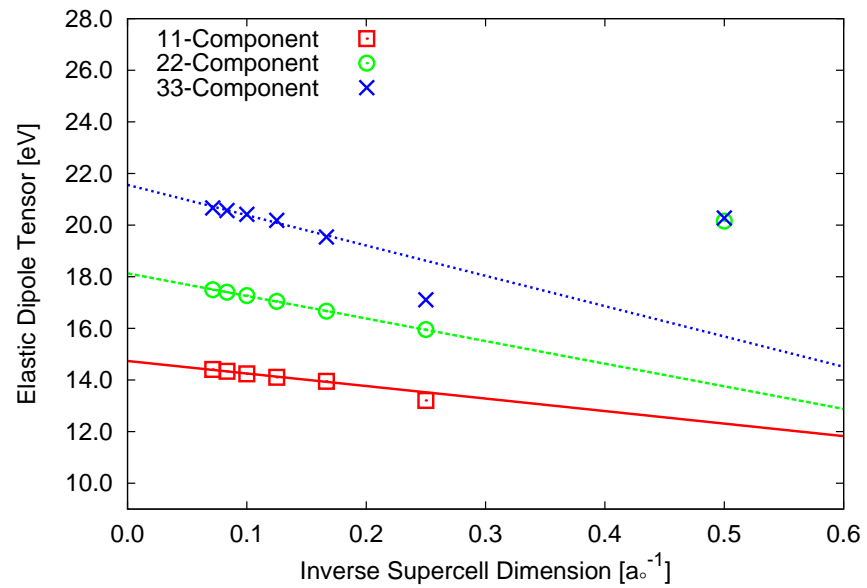

FIGURE 10: Diagonal components of elastic dipole tensor for titanium-oxygen divacancy in strontium titanate. Data show linear convergence with inverse linear dimension of supercell size.

\section{E. Titanium-oxygen divacancy}

As described above in Section III, there are six distinct orientations for the titanium-oxygen divacancy as defined by the $\mathrm{V}_{\mathrm{Ti}}-\mathrm{V}_{\mathrm{O}}$ direction. Moreover, because of the reconstruction, there are in fact two distinct classes of sites within each possible orientation, as distinguished by the rotation state of the octahedron in which the titanium sits. Below, we report results for a [100] defect with the titanium sitting in an octahedron of positive rotation.

The elastic dipole tensor for this defect is

$$
\mathbf{G}_{\mathrm{TiO}}=\left(\begin{array}{rrr}
14.74 & -0.08 & -4.76 \\
-0.08 & 18.13 & -3.87 \\
-4.76 & -3.87 & 21.56
\end{array}\right) \mathrm{eV},
$$

where Figure 10 shows the convergence of the diagonal elements of the above tensor. The reconstructional average gives

$$
\overline{\mathbf{G}}_{\mathrm{TiO}}=\left(\begin{array}{rrr}
14.74 & 0.00 & 0.00 \\
0.00 & 19.84 & 0.00 \\
0.00 & 0.00 & 19.84
\end{array}\right) \mathrm{eV},
$$

with corresponding defect-strain tensor,

$$
\overline{\boldsymbol{\Lambda}}_{\mathrm{TiO}}=\left(\begin{array}{rrr}
3.00 & 0.00 & 0.00 \\
0.00 & 21.68 & 0.00 \\
0.00 & 0.00 & 21.68
\end{array}\right) \AA^{3} .
$$

The defect-strain tensor expresses the tendency of this defect to expand the crystal in all directions, but primarily along the directions orthogonal to the $\mathrm{V}_{\mathrm{Ti}}-\mathrm{V}_{\mathrm{O}}$ axis. When the above tensor is averaged over all six defect orientations (oxygen sites in octahedra surrounding the central titanium site, recalling that the $\mathrm{V}_{\mathrm{Ti}}-\mathrm{V}_{\mathrm{O}}$ axis is direction dependent), the result is a constant tensor with a uniform chemical strain per unit concentration of defect

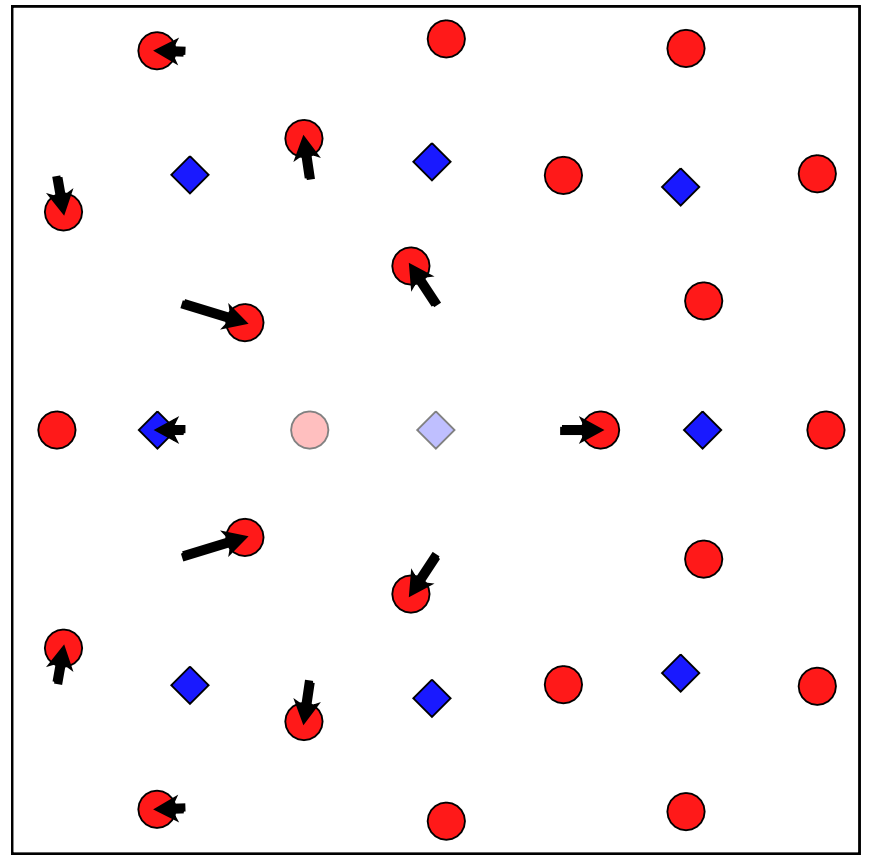

FIGURE 11: Local strain pattern for titanium-oxygen divacancy in the $\hat{e}_{1} \hat{e}_{2}$ plane of strontium titanate: titanium atoms $(\diamond)$, oxygen atoms $(\bigcirc)$, titanium vacancy $(\diamond)$, oxygen vacancy $(\bigcirc)$. Atomic displacements exaggerated by a factor of three for clarity $(\rightarrow)$, displayed for significant in-plane displacements $(>0.1 \AA)$ only.

of $+15.45 \AA^{3}$. Such expansion corresponds to a ratio of chemical strain $\epsilon_{c}$ to the deviation from stoichiometry $\delta$ in $\mathrm{SrTi}_{1-\delta} \mathrm{O}_{3-\delta}$ of $\epsilon_{c} / \delta=+0.260$, indicating a significant tendency for the crystal to expand due to the presence of titanium-oxygen divacancy.

Now that our reconstructional averaging has symmetrized the displacement patterns of neighboring atoms, it is instructive to examine this set of near-field atomic strains, shown in Figure 11. The situation with this divacancy is again more complicated than that of earlier isolated atomic vacancies. There are six atoms in the first shell around the divacancy. The five remaining (the sixth is itself missing) oxygen atoms that are nearest neighbors of the titanium vacancy all move away from that vacancy: the one that is furthest from the oxygen vacancy moves $0.20 \AA$ directly away from the vacancy, while the other four (which are in the $\hat{e}_{2} \hat{e}_{3}$ plane) move $0.23 \AA$ away from the vacancy $\left(0.13 \AA\right.$ along $\hat{e}_{1}$ toward the oxygen vacancy, with the remaining projection of $0.19 \AA$ away from the divacancy either along $\hat{e}_{2}$ or $\hat{e}_{3}$ as dictated by the symmetry). The sixth atom in this first shell is the sole titanium atom that is the nearest neighbor to the oxygen vacancy; it moves directly away from the vacancy by $0.13 \AA$. In the second shell, we identify four of the oxygen atoms that are nearest neighbor to the oxygen vacancy (we already counted the other four nearest-neighbor oxygens above, "assigning" them to the titanium vacancy); these oxygens move $0.33 \AA$ toward the oxygen vacancy $\left(0.31 \AA\right.$ along $\hat{e}_{1}$, 


\begin{tabular}{cr}
\hline Defect & \multicolumn{1}{c}{$\epsilon_{c} / \delta$} \\
\hline $\mathrm{V}_{\mathrm{O}}$ & 0.001 \\
$\mathrm{~V}_{\mathrm{Sr}}$ & 0.030 \\
$\mathrm{~V}_{\mathrm{Ti}}$ & 0.402 \\
$\mathrm{~V}_{\mathrm{SrO}}$ & -0.008 \\
$\mathrm{~V}_{\mathrm{TiO}}$ & 0.260 \\
\hline
\end{tabular}

TABLE III: Individual ratios of chemical strain $\epsilon_{c}$ to stoichiometric defect deviation $\delta$ for different defects as calculated in Section IV

with remaining projection of $0.10 \AA$ either along $\hat{e}_{2}$ or $\hat{e}_{3}$ as dictated by symmetry). Also, in this second shell, we can loosely consider the eight nearest-neighbor strontium atoms to the titanium vacancy, all of which move toward the titanium vacancy: four of these strontium atoms are in a plane that includes the oxygen vacancy, and these move $0.22 \AA$ toward the titanium vacancy $(0.17 \AA$ along $\hat{e}_{1}$, and $0.09 \AA$ along both $\hat{e}_{2}$ and $\hat{e}_{3}$ ); the other four strontium atoms are on the opposite side of the titanium vacancy, and these move significantly toward the vacancy by $0.58 \AA\left(0.38 \AA\right.$ along $\hat{e}_{1}$, and $0.31 \AA$ along both $\hat{e}_{2}$ and $\left.\hat{e}_{3}\right)$. All other atoms move less than $0.20 \AA$.

We again examine connections between the reconstructionally averaged local displacements and the far-field defect-strain tensor. In this case of the titanium-oxygen divacancy, the nearest-neighbor atoms to both vacancies move outward, showing the same behavior as the defect-strain tensor. These atoms also demonstrate larger movements in those directions orthogonal to the $\mathrm{V}_{\mathrm{Ti}}-\mathrm{V}_{\mathrm{O}}$ axis, which conforms with the far-field tensor. The nextnearest neighbors to both vacancies move inward with significant displacements, with those closest to the titanium vacancy moving by almost three times the amount that the nearest-neighbor atoms move. So here we note once more that the far-field strain does not appear to correlate with the largest magnitude displacement, but instead with that of the nearest-neighbor atoms.

\section{DISCUSSION}

The above results for the local strain patterns and detailed defect tensors are now available for direct comparison with diffuse x-ray measurements; however, we are not aware of any such x-ray data to date. Nonetheless, the fully averaged (over both reconstructions and orientations) defect-strain tensors relate directly to measurements which are commonly done of chemical strain as a function of defect concentration. TableIII summaries the results from Section IV for the ratios of chemical strain $\epsilon_{c}$ to stoichiometric defect deviation $\delta$ for all of the defects considered in this study.

Oxygen-vacancy concentration is widely thought to serve a crucial role in the properties of perovskites, 46 is readily varied but difficult to control, 5455 and is experimentally observed to affect chemical strain. ${ }^{[5]}$ Moreover,

\begin{tabular}{cr}
\hline Reference state & $\Delta \epsilon_{c} / \delta$ \\
\hline Bulk & 0.001 \\
$\mathrm{~V}_{\mathrm{Sr}}$ & -0.038 \\
$\mathrm{~V}_{\mathrm{Ti}}$ & -0.142 \\
\hline
\end{tabular}

TABLE IV: Net ratios of chemical strains to stoichiometric defect deviation for oxygen vacancies, referenced against bulk and isolated cation vacancies as appropriate.

cation stoichiometry is also difficult to control $\frac{57 / 58}{5}$ and so it is uncertain whether, as oxygen vacancies are introduced into the crystal, such vacancies bind to cation vacancies or form in isolation. In the former case, where the oxygen vacancies eventually bind to preexisting cation vacancies, the reference configuration should be the crystal containing the cation vacancy. Hence, it is the difference between the chemical strain of the oxygen-cation divacancy and that of the isolated cation vacancy that describes the change in the crystal lattice as a function of varying oxygen-vacancy concentration. In the latter case of isolated vacancies, the bulk crystal is in fact the system into which these vacancies are introduced, and thus the chemical strain as a function of oxygen-vacancy concentration is described precisely by that of the isolated oxygen vacancy in our study. Table IV summarizes the resulting net chemical strain $\Delta \epsilon_{c}$ versus oxygen-vacancy concentration $\delta$.

For the oxygen vacancy, we have the intriguing result that the elastic dipole tensor and corresponding defectstrain tensor almost vanish under orientational averaging. Thus, very little net effect on the lattice can be expected from the presence of isolated oxygen vacancies. Moreover, the large anisotropy of the dipole tensor of the oxygen vacancy and the ease of introduction and high mobility of such vacancies should allow for the control of the population and orientation of oxygen vacancies by applying external stress. (For instance, at $1 \%$ strain, the orientational energy differences from $\overline{\mathbf{G}}_{\mathrm{O}}$ are $66 \mathrm{meV}$, or about 2.6 times room temperature.) Also, such vacancies can be expected to tend to shield internal crystalline stresses that result from materials processing, a fact potentially related to the observed difficulties in controlling the oxygen-vacancy concentration during crystalline growth.

One of the earliest sets of available experimental data on chemical strain due to oxygen vacancies in strontium titanate comes from Yamada and Miller, ${ }^{56}$ who unfortunately found a null result. Nonetheless, that null result places bounds which, in conjunction with our results, allow some conclusions to be drawn. Yamada and Miller varied the oxygen-vacancy concentration over a range from nearly zero up to $3.24 \times 10^{19} \mathrm{~cm}^{-3}(\delta=0.0019$ in $\mathrm{SrTiO}_{3-\delta}$ ), stating that "no volume change upon reduction was assumed," due to the experimental uncertainty of the lattice constant $\left(\Delta a=5 \times 10^{-4} \AA\right)$ in their x-ray diffraction measurements. Their detection limit for chemical strain per stoichiometric defect deviation is 
therefore $\left|\epsilon_{c} / \delta\right|=|(\Delta a / a) / \delta|<0.066$, where $a$ is the cubic lattice constant. From Table IV it is evident that this bound is consistent with either isolated oxygen vacancies or strontium-oxygen divacancies, but is inconsistent with titanium-oxygen divacancies.

The literature also presents studies of chemical strain due to cation non-stoichiometry. Ohnishi et al ${ }^{[59}$ present experimental results on the ratio of chemical strain to deviation from cation stoichiometry for samples grown by pulsed laser deposition. Specifically, they measure the lattice changes for both strontium-rich and strontium-poor strontium titanate. They associate the strontium-rich phase with creation of Ruddlesden-Popper $\underline{60 \mid 61}$ planar faults (extra SrO layers) and the strontium-poor regime with the presence of strontium vacancies, possibly bound into strontium-oxygen divacancies. A leastsquares fit to the results of Ohnishi and coworkers provides a value for $\epsilon_{c} / \delta$ between +0.13 (non-weighted) and +0.14 (weighted by reported experimental uncertainty) for the strontium-rich phase $\left(\mathrm{Sr}_{1+\delta} \mathrm{TiO}_{3}\right)$ and between +0.5 (non-weighted) and +0.8 (weighted by reported experimental uncertainty) for the strontium-poor phase $\left(\mathrm{Sr}_{1-\delta} \mathrm{TiO}_{3}\right)$.

Our calculations of titanium-vacancy chemical strains are not directly relevant to the strontium-rich phase because they do not account for Ruddlesden-Popper planar faults. On the other hand, our results for strontium vacancies, under the interpretation of Ohnishi et al., 59 should be directly relevant to their strontium-poor samples. Table III gives $\epsilon_{c} / \delta=+0.030$ and $\epsilon_{c} / \delta=-0.008$, respectively, for isolated strontium vacancies and bound strontium-oxygen divacancies. However, both of these are an order of magnitude smaller than the observed chemical strain. (We note parenthetically that, while our calculations reflect chemical strains for isolated defects and Ohnishi and coworkers measure strains for relatively high defect densities, Figures 4 and 8 show that trends in our data with increasing defect concentration only tend to reinforce our conclusions.) We remark that our results are consistent with the observation that, within a given structural class, the lattice constants of the titanates are largely insensitive to the nature of the A-site cations. (See data on $\mathrm{A}^{2+} \mathrm{B}^{4+} \mathrm{O}_{3}$ perovskites compiled by Galasso ${ }^{62}$ ) From both the results of our calculations and this general observation, it seems implausible that simple A-site vacancies should produce the measured magnitude of chemical strain. Chemical strains of the magnitude measured by Ohnishi and coworkers more plausibly arise from B-site vacancies or defect complexes associated with such vacancies.

We note that, intriguingly, there is an approximate coincidence between the order of magnitude of our calculated ratio of chemical strain to stoichiometric deviation due to titanium vacancies, $\left|\epsilon_{c} / \delta\right|=0.4$, and the observed values $(+0.5$ and +0.8 , depending upon weighting of fit) for the strontium-poor samples. Given the magnitude of the observed lattice expansion and the fact that only titanium vacancies appear capable of producing an effect of this size, we are led to the intriguing conjecture that, perhaps, the strontium-poor samples exhibit defects that include titanium vacancies and thus a structure more complex than initially thought (simple strontium vacancies or strontium-oxygen divacancies). Clearly, more investigation is needed on this point, specifically as to the nature of the point defects in the strontium-poor samples grown by pulsed laser deposition.

The above measurements by Ohnishi and coworkers 59 were performed on strontium titanate samples deposited by the highly energetic process of pulsed laser deposition $[63$ We have learned of recent results by Brooks et al., 64 that repeat the above measurements on strontium titanate samples grown by molecular-beam epitaxy, which is a lower-energy deposition process $\sqrt[65 \mid 66]{6}$ and thus less prone to the creation of point defects. $\stackrel{63}{ }$ Performing a weighted least-squares fit to the new data of Brooks and coworkers, we find a value for $\epsilon_{c} / \delta$ of $+0.032 \pm 0.019$ in the dilute limit of the strontium-poor regime. This chemical strain ratio, published after our initial calculated prediction was submitted to this journal, shows good agreement with our value of +0.030 , and thereby lends convincing support for our methodology as well as the applicability of empirical shell potentials to calculate reasonable estimates of the chemical strain per stoichiometric defect deviation of vacancies.

\section{SUMMARY AND CONCLUSION}

We have calculated both near- and far-field strains for five defects in reconstructed strontium titanate: isolated oxygen, strontium, and titanium vacancies, as well as strontium-oxygen and titanium-oxygen divacancies. Given the propensity of the crystal for local fluctuations in the reconstruction, we report results both for a particular reconstructed state and as averaged over all possible local reconstructions. The reconstructionally averaged near-field strain results are presented and interpreted in terms of the movement of neighboring shells of atoms at increasing distances from the vacancy or divacancy. We report far-field strain results in terms of both elastic dipole tensors, with and without reconstructional averaging, and associated defect-strain tensors, with reconstructional averaging. Anticipating that far-field effects will necessarily involve contributions from an ensemble of defects, we also present results averaged over all possible orientations of the defect within the bulk crystal. From these averaged tensors, we extract the resultant ratio of chemical strain to stoichiometric defect deviation. Finally, the combination of local and long-range results presented herein will enable determination of x-ray scattering signatures for comparison with experimental results and should further motivate future work on defect mechanics, including the influence of externally imposed strain (such as in heterostructures) on vacancy populations.

For the oxygen vacancy, we find a highly anisotropic 
elastic dipole tensor, with almost perfect cancellation under orientational averaging. This may be correlated with observed difficulties in controlling oxygen concentration and lack of observation of effects of oxygen-vacancy concentration on lattice strain. The anisotropy of this tensor also suggests that oxygen vacancies may provide a mechanism to shield local internal strains and that application of external stress may allow for control of oxygen stoichiometry. From comparison to lattice-strain studies, we identify both isolated oxygen vacancies and bound strontium-oxygen divacancies as consistent with the experimentally observed chemical strain as a function of oxygen-vacancy concentration in strontium titanate.

For cation non-stoichiometry, we find strong indications that the point defects in strontium-poor strontium titanate samples grown by pulsed laser deposition are not simple strontium vacancies or strontium-oxygen divacancies, but likely more complicated defect complexes. Further, we identify indications that titanium vacancies may play a role in these defect complexes. Finally, during the review process, we learned of recent experimental data, from strontium titanate films deposited via molecular-beam epitaxy, that show good agreement with our calculated value of the chemical strain associated with strontium vacancies.

\section{Acknowledgments}

Joel Brock, Lena Fitting Kourkoutis, David Muller, and Darrell Schlom joined us in illuminating discussions on the experimental connections of these calculations. This work was supported by the Cornell Center for Materials Research (CCMR) with funding from the Materials Research Science and Engineering Center (MRSEC) program of the National Science Foundation (Cooperative Agreement DMR 0520404).
1 D. E. Grupp and A. M. Goldman, Science 276, 392 (1997).

2 J. H. Barrett, Phys. Rev. 86, 118 (1952).

${ }^{3}$ K. A. Müller and H. Burkard, Phys. Rev. B 19, 3593 (1979).

4 P. A. Fleury, J. F. Scott, and J. M. Worlock, Phys. Rev. Lett. 21, 16 (1968).

5 J. G. Bednorz and K. A. Müller, Phys. Rev. Lett. 52, 2289 (1984).

${ }^{6}$ F. Gervais, B. Calès, and P. Odier, Mater. Res. Bull. 22, 1629 (1987).

7 R. E. Cohen, Nature 358, 136 (1992).

8 H. Uwe and T. Sakudo, Phys. Rev. B 13, 271 (1976).

9 Y. Moritomo, A. Asamitsu, H. Kuwahara, and Y. Tokura, Nature 380, 141 (1996).

10 A. P. Ramirez, J. Phys.: Condens. Matter 9, 8171 (1997).

11 W. Zhong, D. Vanderbilt, and K. M. Rabe, Phys. Rev. Lett. 73, 1861 (1994).

12 K. A. Müller, W. Berlinger, and E. Tosatti, Z. Phys. B 84, 277 (1991).

13 N.-H. Chan, R. K. Sharma, and D. M. Smyth, J. Electrochem. Soc. 128, 1762 (1981).

14 N. G. Eror and U. Balachandran, J. Am. Ceram. Soc. 65, 426 (1982).

15 J. F. Schooley, W. R. Hosler, and M. L. Cohen, Phys. Rev. Lett. 12, 474 (1964).

16 C. S. Koonce, M. L. Cohen, J. F. Schooley, W. R. Hosler, and E. R. Pfeiffer, Phys. Rev. 163, 380 (1967).

17 H. Unoki and T. Sakudo, J. Phys. Soc. Jpn. 23, 546 (1967).

18 B. Alefeld, Z. Phys. 222, 155 (1969).

19 T. von Waldkirch, K. A. Müller, and W. Berlinger, Phys. Rev. B 7, 1052 (1973).

20 W. Zhong and D. Vanderbilt, Phys. Rev. Lett. 74, 2587 (1995).

21 M. E. Lines and A. M. Glass, Principles and Applications of Ferroelectrics and Related Materials (Clarendon Press, Oxford, UK, 1977).

22 A. Fleet, D. Dale, A. R. Woll, Y. Suzuki, and J. D. Brock, Phys. Rev. Lett. 96, 055508 (2006).

23 M. J. Gillan, J. Phys. C 17, 1473 (1984).
24 B. G. Dick, Jr. and A. W. Overhauser, Phys. Rev. 112, 90 (1958).

25 M. J. Akhtar, Z.-U.-N. Akhtar, R. A. Jackson, and C. R. A. Catlow, J. Am. Ceram. Soc. 78, 421 (1995).

26 P. P. Ewald, Ann. Phys. 369, 253 (1921).

27 T. Darden, D. York, and L. Pedersen, J. Chem. Phys. 98, 10089 (1993).

28 U. Essmann, L. Perera, M. L. Berkowitz, T. Darden, H. Lee, and L. G. Pedersen, J. Chem. Phys. 103, 8577 (1995).

29 M. Deserno and C. Holm, J. Chem. Phys. 109, 7678 (1998).

30 R. A. Buckingham, Trans. Faraday Soc. 54, 453 (1958).

31 M. Born and J. E. Mayer, Z. Phys. 75, 1 (1932).

32 J. E. Lennard-Jones, Proc. Phys. Soc. 43, 461 (1931).

33 J. D. van der Waals, Ph.D. thesis, University of Leiden (1873).

${ }^{34}$ G. V. Lewis and C. R. A. Catlow, J. Phys. C 18, 1149 (1985).

35 C. R. A. Catlow and A. M. Stoneham, J. Phys. C 16, 4321 (1983).

36 M. J. Gillan, Philos. Mag. A 43, 301 (1981).

37 M. J. Gillan, Philos. Mag. A 48, 903 (1983).

38 A. M. Stoneham, J. Phys. C 16, L925 (1983).

39 Y. A. Abramov, V. G. Tsirelson, V. E. Zavodnik, S. A. Ivanov, and I. D. Brown, Acta Crystallogr. B 51, 942 (1995).

40 K. A. Müller, W. Berlinger, and F. Waldner, Phys. Rev. Lett. 21, 814 (1968).

41 E. Pytte and J. Feder, Phys. Rev. 187, 1077 (1969).

42 W. Zhong and D. Vanderbilt, Phys. Rev. B 53, 5047 (1996).

43 M. R. Hestenes and E. Stiefel, J. Res. Natl. Bur. Stand. 49, 409 (1952).

44 E. Polak and G. Ribière, Rev. Fr. Inform. Rech. O. 3, 35 (1969).

45 M. Leslie and M. J. Gillan, J. Phys. C 18, 973 (1985).

46 J. F. Scott and M. Dawber, Appl. Phys. Lett. 76, 3801 (2000).

47 M. Cherry, M. S. Islam, and C. R. A. Catlow, J. Solid State 
Chem. 118, 125 (1995).

48 C. H. Park and D. J. Chadi, Phys. Rev. B 57, R13961 (1998).

49 M. V. Raymond and D. M. Smyth, J. Phys. Chem. Solids 57, 1507 (1996).

${ }^{50}$ L. He and D. Vanderbilt, Phys. Rev. B 68, 134103 (2003).

51 V. C. Lo, J. Appl. Phys. 92, 6778 (2002).

52 S. Kimura, J. Yamauchi, M. Tsukada, and S. Watanabe, Phys. Rev. B 51, 11049 (1995).

53 V. E. Henrich, G. Dresselhaus, and H. J. Zeiger, Phys. Rev. B 17, 4908 (1978).

54 R. J. D. Tilley, J. Solid State Chem. 21, 293 (1977).

${ }^{55}$ K. Szot and W. Speier, Phys. Rev. B 60, 5909 (1999).

${ }^{56}$ H. Yamada and G. R. Miller, J. Solid State Chem. 6, 169 (1973).

57 S. Yamamichi, H. Yabuta, T. Sakuma, and Y. Miyasaka, Appl. Phys. Lett. 64, 1644 (1994).

58 T. R. Taylor, P. J. Hansen, N. Pervez, B. Acikel, R. A.
York, and J. S. Speck, J. Appl. Phys. 94, 3390 (2003).

59 T. Ohnishi, K. Shibuya, T. Yamamoto, and M. Lippmaa, J. Appl. Phys. 103, 103703 (2008).

60 S. N. Ruddlesden and P. Popper, Acta Crystallogr. 10, 538 (1957).

61 S. N. Ruddlesden and P. Popper, Acta Crystallogr. 11, 54 (1958).

62 F. S. Galasso, Structure, Properties and Preparation of Perovskite-Type Compounds (Pergamon Press, Oxford, UK, 1969), pp. 18-19.

63 P. R. Willmott and J. R. Huber, Rev. Mod. Phys. 72, 315 (2000).

${ }^{64}$ C. M. Brooks, L. Fitting Kourkoutis, T. Heeg, J. Schubert, D. A. Muller, and D. G. Schlom, Appl. Phys. Lett. 94, 162905 (2009).

65 B. A. Joyce, Rep. Prog. Phys. 48, 1637 (1985).

66 G. B. Stringfellow, Rep. Prog. Phys. 45, 469 (1982). 